\title{
La Evaluación Del Retour En Interpretación Simultánea Árabe-Español: Transmisión Del Discurso Original
}

\author{
Bachir Mahyub Rayaa, PhD \\ Research group Quality Evaluation in Simultaneous Interpreting (ECIS) \\ -Ref. HUM-560- \\ University of Granada, Spain
}

doi: 10.19044/esj.2017.v13n11p261 URL:http://dx.doi.org/10.19044/esj.2017.v13n11p261

\begin{abstract}
Many reasons have boosted the demand for Arabic-Spanish Simultaneous Interpreting (SI), among others the political and economic interdependence and the social and cultural relations between the Arab and the Spanish-speaking world, as well as the growing interest in the contemporary Arabic world for strategic and geopolitical reasons. Meetings and multilateral conferences have proliferated from the second half of the twentieth century. In addition, Arabic and Spanish are official languages at international organizations like the United Nations or the African Union, and they are present in the private international market of SI.

However, research on the quality of Spanish-Arabic interpreting (AR-SP) remains limited, raising numerous questions on the evaluation and quality control of a profession that enables communication in an increasingly globalized world.

This article, which is part of a wider study analyzing how users of interpreting services evaluate AR-SP SI, focuses on the analysis of two parameters: correct transmission and complete transmission of the Original Speech (OS). For this purpose, we have carried out an empirical study using a questionnaire to survey 25 users of interpreting services, asking them to observe and evaluate AR-SP SI over the course of a 30 minute conference. The data obtained reveals that the users are aware of the concept of quality regards interpreting and, despite not having access to the OS, form an opinion on interpreters and their work. Ultimately, in this article we investigate the elements that influence how users of interpreting services evaluate AR-SP interpreting and the consequences these elements have on the work of the interpreter.
\end{abstract}


Keywords: Assessment, Simultaneous Interpreting, Arabic-Spanish, Retour, Original Speech Transmission.

\section{Resumen}

Numerosos son los factores que han favorecido el auge de la demanda de la interpretación simultánea (IS): la interdependencia política y económica, las relaciones sociales y culturales entre el mundo árabe y el mundo de habla hispana, así como el creciente interés por el mundo árabe contemporáneo por motivos estratégicos y geopolíticos. Esto ha favorecido la proliferación de reuniones, congresos y conferencias bilaterales y multilaterales a partir de la segunda mitad del siglo XX. Además, tanto el árabe como el español son lenguas oficiales de organismos internacionales como las Naciones Unidas o la Unión Africana, y están presentes en el mercado internacional privado de la IS.

Sin embargo, la investigación sobre la calidad de la interpretación árabeespañol (AR-ES) es un terreno aún inexplorado, lo que plantea numerosas incógnitas sobre la evaluación y el seguimiento de calidad de este oficio que hace posible la comunicación en un mundo cada vez más globalizado.

Este artículo, que forma parte de un trabajo más amplio en el que analizamos la evaluación de los usuarios de la IS AR-ES inversa o retour, se centra en el análisis de dos parámetros interconectados: transmisión correcta y transmisión completa del discurso original (DO). Para ello, realizamos un estudio empírico mediante cuestionario, en el que sondeamos la opinión de 25 usuarios. A estos usuarios se les ha pedido visionar y evaluar la IS AR-ES de una conferencia de 30 minutos. Los datos obtenidos revelan que los usuarios de la interpretación son conscientes del concepto de calidad de la interpretación y que, si bien no tienen acceso al DO, sí se forman una opinión sobre la interpretación en sí y sobre el intérprete. En definitiva, en esta contribución indagamos en los elementos que influyen en la evaluación de los usuarios de la interpretación AR-ES y sus consecuencias sobre el trabajo del intérprete.

Palabras clave: Evaluación, Interpretación simultánea, Árabe-Español, Retour, Transmisión del discurso original.

\section{Introducción teórica general}

La práctica de la interpretación, como ejercicio de intermediación entre dos personas que no comparten el mismo código lingüístico se remonta necesariamente a los albores de la historia de la humanidad. En este sentido, se ha dicho que la interpretación es uno de los oficios más antiguos, muy anterior a la traducción escrita, dado que la lengua oral surge mucho antes que la escritura (Haensch, 1965). Sin embargo, la comunidad investigadora 
actual coincide en ubicar los inicios de la profesionalización de la interpretación de conferencias (IC), tal y como la conocemos hoy en sus dos vertientes consecutiva y simultánea, después de la I Guerra Mundial, en el periodo de entreguerras (Baigorri, 2014: 133-164). La organización de su enseñanza como disciplina académica comienza a impartirse de forma reglada en centros europeos como la Escuela de Ginebra (ETI) o el ESIT de París a finales de los cincuenta del siglo XX (Martin, 1999 e Iglesias, 2007, entre otros).

Como es lógico, la evaluación y el seguimiento de la calidad de este oficio que hace posible la comunicación en un mundo cada vez más globalizado es de grave importancia. Este ha sido precisamente el objetivo por el que se inició la actividad investigadora en el ámbito de la calidad de la interpretación. En 1986, Bühler publicaría su primer trabajo acerca de la opinión de los intérpretes sobre los posibles factores que determinan la calidad de la interpretación. Si bien varios son los trabajos que ya habían abordado algunos aspectos de la calidad de la interpretación (Gerver, 1974; Anderson, 1979 y Gile, 1983, entre otros), es a raíz de este trabajo de Bühler (1986) cuando se aprecia una fijación de los primeros parámetros de calidad, así como una continuidad por las mismas líneas de investigación, sondeando no solo las opiniones de los intérpretes, sino también las del público y los organizadores de eventos. Autoras como Kurz (2001), Pradas Macías (2004) o Collados Aís et al. (2007) realizan un exhaustivo repaso de la investigación empírica sobre la calidad de la interpretación hasta la actualidad. En los últimos años, este ámbito ha visto ensanchadas sus fronteras al incorporar nuevas combinaciones lingüísticas y abordar nuevos enfoques de estudio. En esta línea se inserta nuestro trabajo, que se centra en la evaluación de los usuarios de la interpretación AR-ES, en el mismo sentido que inició Kurz (1989) y la siguieron Gile (1990), Kopczynski (1994), entre otros, en combinaciones lingüísticas que no comprendían el árabe.

A pesar de que la interdependencia política y económica, las relaciones sociales y culturales entre el mundo árabe y el mundo de habla hispana, así como el creciente interés por el mundo árabe contemporáneo por motivos estratégicos y geopolíticos favorecieron, a partir de la segunda mitad del siglo XX, la proliferación de reuniones, congresos y conferencias multilaterales, y, por ende, una inusitada demanda de la interpretación ARES en todas sus formas, la investigación en el ámbito de la calidad de la interpretación, ya sea de conferencia o en los servicios públicos, y su evaluación en este par de lenguas sigue siendo un terreno aún inexplorado. En nuestra revisión exhaustiva de la bibliografía, solo hemos hallado dos trabajos relacionados con la evaluación de la calidad de la interpretación ARES: Barbato (2014), un artículo que analiza la credibilidad de la voz del intérprete de Rabei Osman Sayad “El Egipcio” en el juicio del 11-M; y 
Mahyub Rayaa (2013), que realiza un estudio sobre la práctica de la interpretación simultánea AR-ES, en el que dedica un apartado al análisis de los factores que más inciden en la calidad de la interpretación en esta combinación lingüística.

Además de la escasa literatura previa y la necesidad de investigación empírica en interpretación AR-ES, uno de los motivos que nos ha llevado a realizar este trabajo es el hecho de que, en España y en el mundo árabe, la interpretación conferencia AR-ES la ejercen casi siempre intérpretes de origen árabe no nativos de español (Mahyub Rayaa, 2015: 143) ${ }^{46}$. Además, en la práctica profesional, todo indica que la unión de varios factores como la demanda inesperada, el carácter minoritario de la lengua árabe, al menos en España, y la falta de intérpretes con español como lengua materna, entre otros, han obligado a cubrir la demanda del mercado con cabinas biactive, esto es, que los mismos intérpretes vierten al árabe y al español. Esta afirmación viene refrendada por el hecho de que el 91,6\% de los intérpretes de conferencias AR-ES afincados en España afirma que ejerce la IS directa e inversa (Mahyub Rayaa, 2015: 198). No obstante, el español es la lengua $B^{47}$ del $\mathbf{8 7 . 5 \%}$ de ellos. Esta realidad no parece exclusiva ni del panorama español ni de la combinación AR-ES, sino que, a la luz de las conclusiones alcanzadas por Al-Salman y Al-Khanji (2002: 608-624), parece que se da también en interpretación simultánea del árabe al inglés.

En términos prácticos, encontramos que de la cabina AR-ES depende, además del público español, la interpretación a las otras lenguas, dado que el resto de intérpretes -en encuentros multilingües- tomará el relé español de la cabina AR-ES para poder verter a sus respectivas lenguas meta. Por lo tanto, observamos que cuando se emplea el árabe en un acto multilingüe en España, casi todos los oyentes dependen de la interpretación que la cabina árabe haga al español. Por todos estos motivos consideramos relevante para el ámbito académico y profesional analizar cómo perciben los usuarios una IS AR-ES realizada por un intérprete no nativo de español, así como indagar hasta qué punto los resultados que arroje este estudio pueden ser de utilidad para la decencia y la práctica profesional de la interpretación.

A pesar de que autores como Gile (1983), Collados Aís (1998), Gracia García (2003), entre otros, señalan que es imposible que los usuarios puedan juzgar objetivamente la fidelidad de una interpretación sin tener acceso al discurso original (DO), o que su capacidad de evaluación del contenido de la

\footnotetext{
${ }^{46}$ De los 16 intérpretes de conferencia AR-ES que el autor entrevistó, solo dos poseían el español como lengua A (materna).

47 Según la clasificación de la Asociación Internacional de Intérpretes de Conferencia (AIIC): "A language other than the interpreter's native language, of which she or he has a perfect command and into which she or he works from one or more of her or his other languages".
} 
interpretación es restringida, varios son los autores (Kurz, 2001; Pradas Macías, 2004; Collados Aís et al., 2007; entre otros) que han confirmado que los usuarios siempre opinan sobre el contenido de la interpretación aunque desconozcan la lengua origen o no tenga acceso al DO, lo que unido a su papel de clientes finales del encargo de la interpretación le otorga una relevancia considerable al análisis de los criterios en que estos usuarios basan su evaluación.

En esta contribución, que forma parte de un trabajo más amplio, nos centraremos en el análisis de la evaluación que los usuarios de la interpretación realizan de dos parámetros de contenido interconectados: transmisión correcta del DO y transmisión completa del sentido original. El parámetro "transmisión correcta del DO” es probablemente el más importante cuando se trata de evaluar la calidad de una interpretación (Fernández Sánchez et al., 2007: 89-104). No obstante las reservas de varios autores al respecto de la capacidad real del público de evaluarlo objetivamente (Gile, 1995 y Collados Aís, 1998), lo cierto es que a este parámetro, entendido como la fidelidad del intérprete hacia el DO o lealtad al mensaje original, tanto usuarios como intérpretes le otorgan una gran importancia. Según Bühler (1986), es el parámetro de mayor importancia para el 96\% de los intérpretes que esta autora sondeó en su trabajo. El segundo parámetro, el de “transmisión completa del sentido original”, está relacionado de forma directa con el primero. De hecho, diversos son los estudios que los han analizado como variables dependientes entre sí ( $c f$. Pradas Macías et al., 2007: 109-114). En el ámbito de la interpretación se ha asociado este parámetro (Altman, 1994 y Falbo, 1998) a la presencia de omisiones de diversa índole en la producción del intérprete. En los estudios de expectativas de calidad de los usuarios de la interpretación, este parámetro goza de una incidencia media alta, sin embargo, si se asocia a la transmisión completa y a la cohesión lógica, que ocupan los puestos más importantes según usuarios e intérpretes, su incidencia aumenta notablemente (Pradas Macías et al., 2007: 113).

\section{Objetivos}

Este trabajo pretende, de forma general, realizar una primera aproximación a la evaluación de los usuarios de la IS AR-ES, en particular del contenido de la interpretación. Más en concreto, pretende alcanzar los siguientes objetivos específicos:

- Averiguar si los usuarios de la interpretación, sin tener acceso al DO, entran a juzgar si su trasmisión por parte del intérprete es correcta o no.

- En caso de hacerlo, averiguar qué factores inciden en su evaluación. 
- Averiguar si los usuarios de la interpretación, sin tener acceso al DO, entran a juzgar si el intérprete ha sabido trasmitir todo el sentido original o no.

- En caso de hacerlo, averiguar qué factores inciden en su evaluación.

- Averiguar en qué medida influye en la evaluación de los usuarios de la interpretación el hecho de que el intérprete no sea nativo de español.

\section{Material y método}

Para la realización de este estudio hemos empleado la grabación en vídeo de una interpretación AR-ES real ${ }^{48}$, de 30 minutos de duración. Se trata de la presentación de la traducción de la novela $A$ escondidas, a cargo de su propio autor Sonallah Ibrahim (Egipto). El acto de presentación fue celebrado en la Casa Árabe (Madrid), el 13 de junio de 2013. La interpretación de ese acto al español fue realizada íntegramente por un solo intérprete, que cuenta con más de 20 años de experiencia en este oficio. Nos hemos inclinado por un acto de temática literaria porque consideramos que es un ámbito que requiere del dominio de un registro lingüístico alto en español, especialmente cuando en el mismo acto se ha leído un fragmento de la novela y el intérprete optó por verterlo al español sin contar con la traducción oficial.

Por otra parte, elaboramos un cuestionario de nueve preguntas dividido en dos apartados principales. Uno introductorio sobre el perfil de los encuestados (sexo, edad, lenguas y si cursa o ha cursado estudios de Traducción e Interpretación); y otro en el que se incluyen las nueve preguntas sobre el contenido de la interpretación: cuatro semicerradas y cinco abiertas. Dado que en este trabajo pretendemos realizar una primera aproximación a un campo aún inexplorado, en todas las preguntas, a excepción de la última, hemos contemplado con un campo de respuesta libre habilitado para que el encuestado pueda justificar su respuesta. De este modo, obtendríamos información cuantitativa muy valiosa para un estudio de esta índole.

El cuestionario (véase en el anexo) abre y cierra con una pregunta general acerca de la evaluación global de la interpretación. La primera es de respuesta múltiple y con ella pretendemos obtener datos cualitativos. Hemos considerado que empezar con una pregunta de este tipo nos permitiría captar las primeras impresiones del usuario de la interpretación, antes de entrar en los pormenores de su evaluación. En la última pregunta, sin embargo, queríamos obtener datos cuantitativos, esto es, una calificación numérica del 1 al 10.

48 El acto con interpretación está disponible en: https://www.youtube.com/watch?v=607dVVxoQPA [Última consulta: 20/03/2017]. 
El sondeo a los sujetos participantes en el estudio se realizó de forma individual en el marco geográfico de las Facultades de Traducción e Interpretación y la de Filosofía y Letras, ambas de la Universidad de Granada (UGR). Los pasos a seguir con cada participante han respetado escrupulosamente el siguiente orden: (i) se le ha mostrado el vídeo a cada participante sin proporcionarle ningún detalle de la encuesta, con el objetivo de no contaminar su criterio. Inmediatamente después de visionar el vídeo, (ii) se le solicitó responder al cuestionario lo más detalladamente posible, explicándole brevemente el objetivo del mismo y los parámetros que recoge. Para que los resultados sean mínimamente representativos, nos fijamos como objetivo una muestra de entre 20 y 30 encuestados. Finalmente, recogimos 25 encuestas válidas, cuyas respuestas volcamos en una hoja de cálculo de Excel para su posterior análisis cuantitativo y cualitativo.

\section{Sujetos}

Como resultado de la localización geográfica del estudio, los 25 participantes en este estudio son o han sido estudiantes de la UGR. A juzgar por su perfil lingüístico, todos ellos hablan árabe (como lengua A o B) o alguna de sus variantes dialectales, además de tener el español como lengua activa (A o B). Exactamente, el 80\% (20) indicaron que tenían el español como lengua A y el árabe como B, mientras que el 20\% restante (5), afirmó tener el orden inverso. El 84\% (21) de los participantes realizaba o había realizado en el momento del estudio el último curso del Grado en Traducción e Interpretación o el Máster en Traducción Profesional, ambos de la UGR. El 16\% restante (4) cursaba el Grado en Estudios Árabes e Islámicos (UGR). El promedio de edad de los encuestados es de 25,5 años, con una desviación estándar de +/-2,3 años. El 68\% (17) son mujeres, mientras que el 32\% restante (8) son hombres.

\section{Resultados}

A continuación, presentamos los resultados obtenidos en el cuestionario según cada parámetro. Como cierre de este apartado, presentamos los resultados de la evaluación global de los usuarios de la interpretación.

\section{Transmisión correcta del discurso original}

En respuesta a la pregunta cómo calificaría la transmisión del mensaje por parte del intérprete, observamos que la gran mayoría de los usuarios (84\%) indica que es correcta, muy lejos del siguiente grupo (12\%), a los que les parece poco correcta. En la siguiente gráfica se puede apreciar la diferencia. 


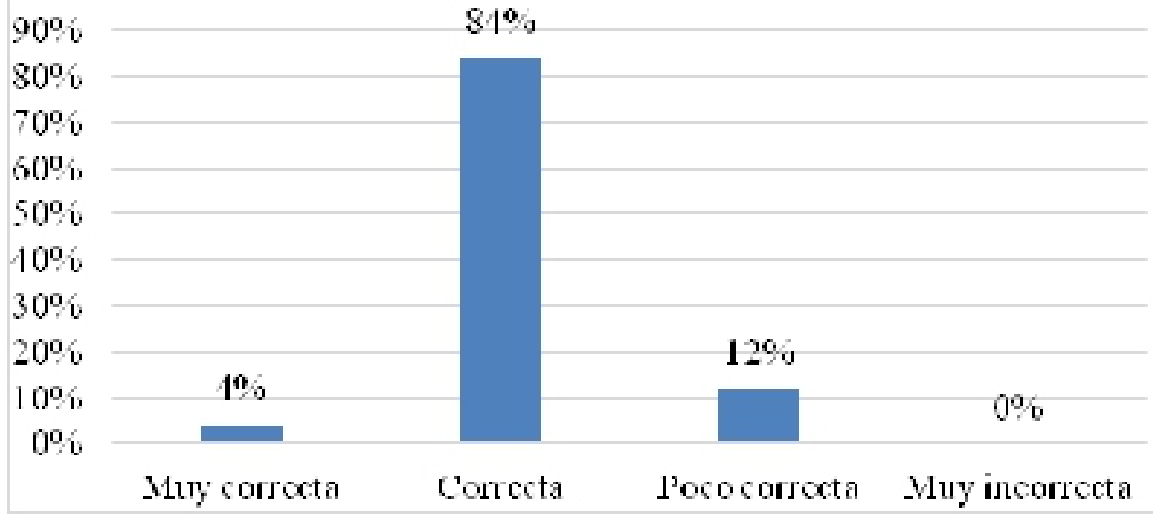

Gráfico 1: Evaluación de la transmisión del DO.

En el campo de respuesta complementaria, los usuarios que lo han empleado indican:

\begin{tabular}{c|c|c}
\hline Usuario & Respuesta & Justificación \\
\hline 001 & Correcta & Porque utilizaba las palabras adecuadas. \\
\hline 002 & Muy correcta & Se ha entendido en todo momento lo que el escritor nos \\
quería transmitir.
\end{tabular}

Tabla 1: Justificación de respuesta sobre transmisión del DO. 


\section{Transmisión completa del sentido original}

En la siguiente pregunta, quisimos averiguar si los usuarios creen que el intérprete ha sabido hacer llegar todo el sentido del mensaje original. El porcentaje de usuarios que responde afirmativamente, si bien disminuye ligeramente con respecto a la pregunta anterior, sigue acaparando la mayoría con el $80 \%$ de los encuestados. Por su parte, se aprecia una ligera subida en el porcentaje de usuarios que consideran que el intérprete no ha sabido hacer llegar todo el sentido del mensaje original (20\%).

Los usuarios que han empleado el campo de respuesta complementaria indican:

\begin{tabular}{|c|c|c|}
\hline Usuario & Respuesta & Justificación \\
\hline 001 & No & $\begin{array}{c}\text { Porque en ocasiones hacía interpretaciones literales que } \\
\text { provocaban la pérdida parcial del sentido. }\end{array}$ \\
\hline 002 & Sí & Creo que concordaba en todo momento con el contexto. \\
\hline 003 & Sí & Es un mensaje correcto en términos generales. \\
\hline 004 & Sí & Aunque no en su totalidad. \\
\hline 005 & Sí & Pero se perciben dudas o inseguridades. \\
\hline 008 & Sí & $\begin{array}{l}\text { Porque en todo momento ha seguido al orador y no se ha parado } \\
\text { a buscar alguna palabra. }\end{array}$ \\
\hline 010 & Sí & $\begin{array}{c}\text { Casi todo el sentido, solo hay que añadir algunos matices. Suele } \\
\text { ocurrir. Se trata de una interpretación simultánea. }\end{array}$ \\
\hline 011 & No & Es poco fluida lo que hace perder la concentración. \\
\hline 012 & Sí & $\begin{array}{c}\text { Aunque repito lo dicho en la respuesta anterior: no he oído el } \\
\text { mensaje original. }\end{array}$ \\
\hline 013 & Sí & Aunque en algunas partes se aprecia una cierta falta de sentido. \\
\hline 016 & Sí & Parece muy creíble a primera vista. \\
\hline 019 & No & $\begin{array}{l}\text { Porque creo que en la interpretación siempre hay pérdidas. } \\
\text { Siempre hay que hacer llegar el } 70 \% \text { como mínimo del mensaje } \\
\text { original. }\end{array}$ \\
\hline 020 & No & Creo que todo no. \\
\hline 022 & Sí & $\begin{array}{c}\text { Creo que el discurso original era fácil y por eso ha sabido hacer } \\
\text { llegar el sentido original. }\end{array}$ \\
\hline 023 & Sí & Lo ha hecho de manera clara y sin dudar. \\
\hline 024 & No & Lo ha intentado pero ha perdido partes del mensaje original. \\
\hline 025 & Sí & $\begin{array}{l}\text { Aunque en algunas partes ha desviado el sentido que quería } \\
\text { transmitir el orador. }\end{array}$ \\
\hline
\end{tabular}

Tabla 2: Justificación de respuesta sobre transmisión del sentido original.

\section{Evaluación global de la interpretación}

Con el fin de recoger lo más nítidamente posible las impresiones de los usuarios, lo primero por lo que se les preguntó fue por su evaluación global de la misma: 


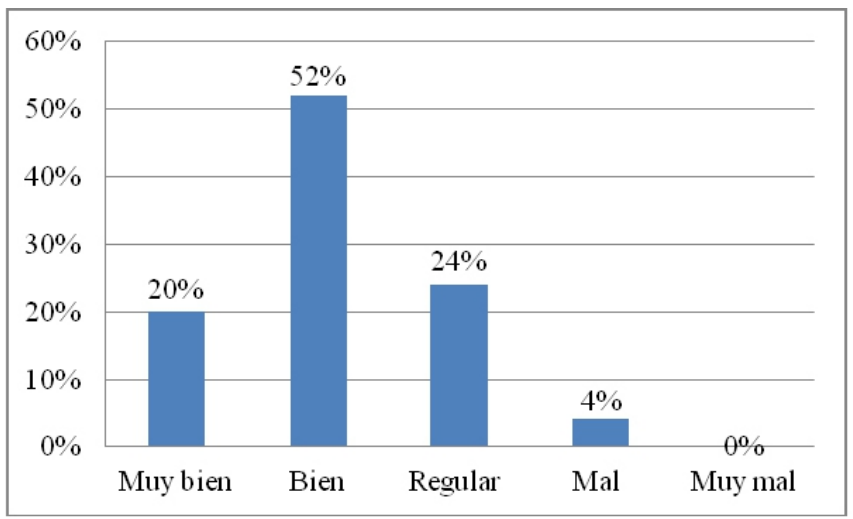

Gráfico 2: Valoración inicial de la interpretación

Los usuarios que han ampliado su respuesta opinan:

\begin{tabular}{|c|c|c|}
\hline Usuario & Respuesta & Justificación \\
\hline 001 & Bien & $\begin{array}{c}\text { Porque al intérprete le faltaban palabras y las completaba con } \\
\text { coletillas no muy usadas en castellano, tipo y “todo eso”, además de } \\
\text { la mala pronunciación de algunas palabras. }\end{array}$ \\
\hline 002 & Bien & $\begin{array}{l}\text { El intérprete ha traducido de manera clara toda la ponencia del } \\
\text { escritor. }\end{array}$ \\
\hline 005 & Bien & $\begin{array}{c}\text { A excepción de algunos problemas de vocalización, la interpretación } \\
\text { es bastante fluida y se entiende perfectamente. }\end{array}$ \\
\hline 006 & Regular & $\begin{array}{l}\text { En algunos casos el intérprete se atrancaba, y no es preciso en su } \\
\text { interpretación. }\end{array}$ \\
\hline 008 & Bien & Transmite el sentido e intenta seguir al orador como puede. \\
\hline 009 & Bien & $\begin{array}{l}\text { A pesar de ciertas redundancias y repeticiones en el discurso, la } \\
\text { interpretación fue buena y coherente en general. }\end{array}$ \\
\hline 010 & Bien & $\begin{array}{l}\text { Ha transmitido casi todo el mensaje, pero se puede mejorar. Para } \\
\text { evitar la confusión, debería mejorar algunas equivalencias. }\end{array}$ \\
\hline 011 & Regular & Mala estructura en lengua española. \\
\hline 012 & Regular & $\begin{array}{l}\text { Calcos del árabe, aunque llega el mensaje. Voz monótona. Dudas que } \\
\text { le restan credibilidad. }\end{array}$ \\
\hline 014 & Bien & Buena interpretación con ciertos problemillas en español. \\
\hline 016 & Bien & $\begin{array}{l}\text { Porque iba muy sincronizado con la persona que estaba hablando y se } \\
\text { podía seguir muy bien. }\end{array}$ \\
\hline 017 & Muy bien & $\begin{array}{c}\text { Comprensible y coherente a pesar de que en algunos casos se podría } \\
\text { perfeccionar más. }\end{array}$ \\
\hline 018 & Bien & $\begin{array}{c}\text { No ha sido muy buena porque se notaba que el intérprete no se había } \\
\text { preparado el encargo. }\end{array}$ \\
\hline 019 & Muy bien & $\begin{array}{c}\text { Nadie es perfecto pero en general me pareció una interpretación } \\
\text { bastante satisfactoria, ya que reúne los requisitos de fluidez, acento } \\
\text { claro y voz agradable. }\end{array}$ \\
\hline 020 & Regular & Hay errores. \\
\hline 021 & Bien & $\begin{array}{c}\text { La interpretación ha sido muy buena pero tiene el acento muy } \\
\text { marcado. }\end{array}$ \\
\hline 024 & Bien & $\begin{array}{c}\text { Ha entendido la mayor parte del mensaje transmitido por el } \\
\text { conferenciante. }\end{array}$ \\
\hline 025 & Mal & $\begin{array}{l}\text { Es poco coherente y muy literal. Falta de atención, capacidad de } \\
\text { procesamiento, transmisión lenta y fallos estilísticos en la producción } \\
\text { del mensaje. }\end{array}$ \\
\hline
\end{tabular}

Tabla 3: Justificación de la valoración inicial. 
Como cierre del cuestionario, a modo de control, volvimos a realizar la misma pregunta, pero esta vez pedíamos una calificación numérica del 1 al 10 (donde 1 es “muy mal” y 10 “muy bien”). La calificación media de los usuarios es de 7,36 con una desviación estándar de +/-1,22. En el siguiente gráfico se puede apreciar la calificación según cada usuario:

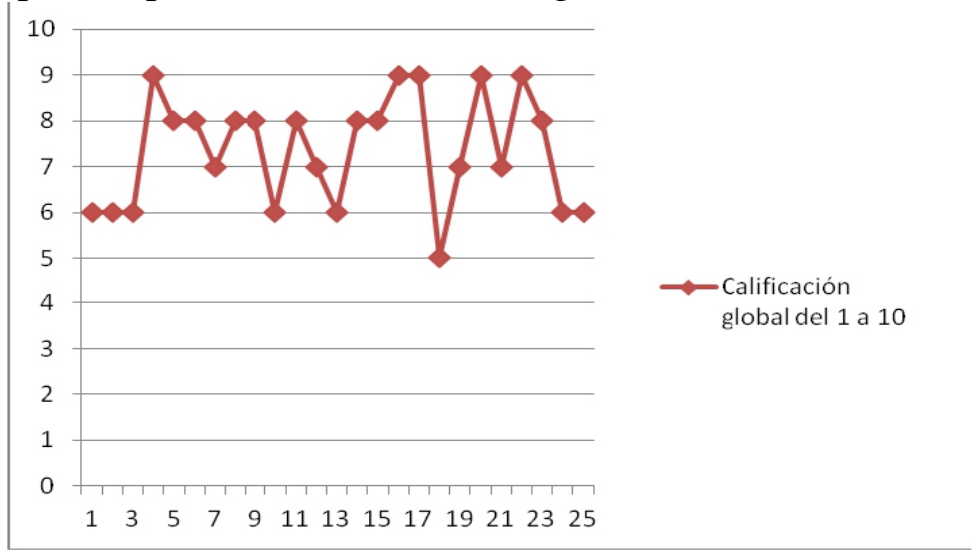

Gráfico 3: Calificación numérica de la interpretación según usuario.

\section{Análisis y discusión}

Procederemos a continuación al análisis y discusión de los resultados obtenidos. En aras de una mayor claridad expositiva, mantendremos la división empleada en el epígrafe anterior.

\section{Transmisión correcta del discurso original}

La gran mayoría de los usuarios (84\%) evalúa la transmisión del DO como correcta, muy lejos de los que la consideran poco correcta (12\%). No obstante, si nos detenemos a analizar los factores en que los usuarios basan su evaluación de este parámetro, observamos una notable heterogeneidad de razones, propia de la subjetividad de cada sujeto (véase tabla 1). El grueso de los usuarios esgrime razones relacionadas con la forma del discurso producido por el intérprete: "uso de las palabras adecuadas" (usuario 001), "posee fluidez y tono de voz correcto" (003), "transmite seguridad y confianza” (005), "uso de coloquialismos” (014), "usa un léxico y estilo pobres" (025). Estos datos refrendarían las conclusiones alcanzadas por Collados Aís (1998), Garzone (2003), y Pradas Macías (2003), entre otros, acerca de la incidencia de los parámetros de forma sobre los de fondo. Esto supone que ciertos aspectos de forma, como es el caso de la fluidez, pueden inducir consciente o inconscientemente a los usuarios de la interpretación a evaluarla positiva o negativamente.

Sin embargo, en este apartado varios son los usuarios que basan su opinión en elementos como la coherencia y el sentido del discurso del 
intérprete (002, 008, 013, 015, entre otros), lo que también confirmaría que no solamente observan la forma sino también el fondo. En este sentido, llama la atención que varios usuarios señalen que la interpretación es deficiente en alguno de sus extremos, si bien la han calificado como correcta (véase los usuarios 004, 007, 014, 020, entre otros), lo cual podría indicar que las deficiencias observadas no implican para estos usuarios una evaluación negativa de la interpretación en su conjunto. Esto apuntaría en la misma línea que Gracia García (2003: 304) cuando dice que, "si se sondeara la opinión del público oyente de una interpretación, probablemente ciertos errores gramaticales pasen desapercibidos siempre y cuando el intérprete logre transmitir el mensaje fundamental y los principales argumentos con claridad".

De todos los usuarios que califican la transmisión del DO como correcta, solamente uno (véase tabla 1, usuario 012) argumenta "creo que ha transmitido el mensaje original aunque, al no poder compararlo con el original, no puedo saberlo del todo". Incluso en este caso, el usuario tampoco se abstiene de evaluar el fondo de la interpretación.

De los resultados obtenidos se colige una primera cuestión importante, a saber: que todos los sujetos acceden a evaluar el contenido de la interpretación sin haber tenido acceso al discurso original, lo que respondería a uno de los objetivos que nos marcamos en este trabajo, al tiempo que refrenda las conclusiones de otros autores (Fernández Sánchez et al., 2007).

\section{Transmisión completa del sentido original}

La gran mayoría de los usuarios (80\%) opina que el intérprete sí ha sabido trasmitir todo el sentido del mensaje original, frente a un $20 \%$ que opina que no lo ha hecho (véase gráfico 2), esto es, un $8 \%$ más que los que calificaban en la pregunta anterior la interpretación como "poco correcta". Si comparamos los resultados obtenidos en este epígrafe con el anterior, observamos aquí que son más los usuarios que motivan sus respuestas en la coherencia y el sentido de la producción del intérprete (véase tabla 2, usuarios 002, 003, 008, 010 y 016), especialmente en el caso de los que responden afirmativamente. Estos resultados reforzarían las afirmaciones de (Gracia García, 2003: 303): “el oyente sí está en condiciones de evaluar algunos aspectos del contenido a partir de sus conocimientos sobre el tema, como la coherencia del mensaje, el uso correcto de la terminología específica o el estilo".

Los que responden negativamente, sin embargo, se inclinan más por aspectos formales como la literalidad del intérprete (001) o la falta de fluidez en su interpretación (011). En este sentido, el parámetro fluidez/pausas vuelva a sobresalir como justificación de si el intérprete ha transmitido el DO íntegramente o no. Esto nos devuelve necesariamente a las investigaciones 
que asocian este parámetro a la presencia de omisiones en la producción del intérprete (Altman, 1994 y Fabro, 1998, entre otros). A este respecto, quizás el uso del formato vídeo en nuestro estudio pudo tener alguna influencia, puesto que el usuario puede cotejar las pausas del intérprete con la imagen del orador, aunque convienen aclarar que esto no signifique obligatoriamente que la interpretación sea incorrecta o tenga más omisiones.

Al igual que en el epígrafe anterior, hay usuarios que, si bien consideran que el intérprete sí ha sabido trasmitir todo el sentido del mensaje original, matizan en el campo de respuesta complementaria que esa transmisión es susceptible de mejora (véase los usuarios 004, 005, 010, 012, 013 y 025). En este sentido, conviene señalar que buena parte de los usuarios se muestra coherente con sus respuestas anteriores, lo que le aporta a credibilidad a los resultados obtenidos.

\section{Evaluación global de la interpretación}

La primera valoración de los usuarios de la interpretación (véase gráfico 2) se inclina mayoritariamente por el "bien” (52\%) o "muy bien” (20\%), lo que sumado representa el $72 \%$ de los usuarios, mientras que a casi una cuarta parte del público (24\%) le parece regular. Si analizamos las respuestas complementarias de aquellos usuarios que las han aportado (véase tabla 3), volvemos a encontrarnos con una alta heterogeneidad de criterios. Si bien los usuarios se mantienen coherentes en sus respuestas, los hay que indican como aspectos negativos: un acento marcado en español, mala estructura, literalidad, mal uso del español, falta de fluidez e incoherencia, etc.; y los hay que resaltan aspectos positivos: claridad del mensaje, fluidez, coherencia, voz agradable, etc. Como se puede apreciar, en casi todos los casos destaca la fijación de los usuarios por los aspectos formales de la producción del intérprete. Por otro lado, llama la atención, al igual que en apartados anteriores, que algunos usuarios otorgan un "bien” pero alegan en su valoración deficiencias en la producción del intérprete (001, 005, 009, 010 y 014). La repetición de este hecho nos lleva a resaltar la relatividad de las respuestas aportadas por los usuarios.

En la calificación numérica global se mantiene la coherencia observada en los apartados anteriores, por lo que consideramos que esta pregunta cumple con su cometido de control. El grueso de las calificaciones de los usuarios se agrupa alrededor del notable, con una media de 7,36 sobre 10 con una desviación estándar de +/-1,22. Esto confirma la tendencia de los dos epígrafes anteriores y demuestra que el público, a pesar de su subjetividad, la relatividad de sus impresiones, o incluso su arbitrariedad (Gile 1995), es coherente en su opinión sobre la interpretación analizada. 


\section{Conclusion}

Vistos los objetivos que nos marcamos al inicio de este trabajo, los resultados obtenidos y su posterior análisis y discusión, podemos concluir que:

- Desde el punto de vista metodológico, los resultados obtenidos son coherentes y lógicos. Asimismo, el cuestionario, como método de investigación, ha sido fundamental para alcanzar los objetivos trazados.

- Los usuarios de la interpretación que han participado en este estudio acceden a evaluar los dos parámetros de transmisión correcta y transmisión completa del DO sin tener acceso a la versión original.

- No obstante, la evaluación que hacen los usuarios del contenido de la interpretación es subjetiva y superficial, en línea con Gile (1995), si bien estos usuarios se muestran coherentes y constantes en sus argumentos.

- Los aspectos en que se basa la evaluación de los usuarios de la interpretación son principalmente formales (estructura en español, literalidad, vocabulario, acento, etc.) y no verbales (fluidez, pausas, confianza, agradabilidad de la voz, etc.). Esta conclusión refrenda a Collados Aís (1998), Garzone (2003) y Pradas Macías (2003), entre otros.

- Los usuarios que más se aproximan a la evaluación del contenido de la interpretación basan su criterio en la coherencia y cohesión de la producción del intérprete.

- El hecho de que el intérprete no sea nativo de español no tiene una incidencia significativa en la evaluación de los usuarios de la interpretación. Si bien varios usuarios lo señalan como una deficiencia, esto no se refleja su valoración global.

- A juzgar por el perfil lingüístico del alumnado de interpretación de árabe, así como por el de los intérpretes profesionales AR-ES (Mahyub Rayaa 2015, 142-144), las conclusiones alcanzadas en este trabajo podrían ser de utilidad para la formación de nuevos intérpretes, así como para la práctica profesional de la interpretación AR-ES.

Por todo ello, concluimos que se han conseguido los objetivos iniciales de este trabajo. No obstante, dado el carácter exploratorio del estudio y las características de la población participante, habría que profundizar más en esta línea de investigación. Convendría estudiar otros parámetros de forma y contenido, y aplicar el mismo estudio con población nativa de español que desconozca la lengua árabe. Los resultados obtenidos podrían cotejarse con los de este estudio con el fin de averiguar si las 
variables de los usuarios tienen alguna incidencia en la evaluación de la misma interpretación.

\section{References:}

1. Al-Salman, S. y Al-Khanji, R. (2002). “The Native Language Factor in Simultaneous Interpretation in an Arabic/English Context”, Meta 47: 4, 607-626.

2. Altman, J. (1994). "Error analysis in the teaching of simultaneous interpreting: A pilot study”. En Lambert, S. y Moser-Mercer, B. (eds.), 25-38.

3. Anderson, R.B.W. (1979). "Interpreter roles and interpretation situations”. En Gerver, D. y Sinaiko, H.W. (eds.), 217-230.

4. Baigorri Jalón, J. (2014). From Paris to Nuremberg. The birth of conference interpreting. Ámsterdam/Filadelfia: John Benjamins.

5. Barbato, L. (2014). "La credibilidad de la voz del intérprete en la administración de justicia (Estudio de caso de la voz del intérprete de Rabei Osman Sayad 'El Egipcio' en el juicio del 11-M)”, Transfer 9: 1-2, 127-149.

6. Bühler, H. (1986). "Linguistic (semantic) and extralinguistic (pragmatic) criteria for the evaluation of conference interpretation and interpreters". Multilingua 5: 4, 231-235.

7. Collados Aís, Á. (1998). La evaluación de la calidad de la interpretación. La importancia de la comunicación no verbal. Granada: Comares.

8. Collados Aís, Á. et al. (eds.) (2003). La evaluación de la calidad en interpretación simultánea: decencia y profesión. Granada: Comares.

9. Collados Aís, Á. et al. (eds.) (2007). La evaluación de la calidad en interpretación simultánea: parámetros de incidencia. Granada: Comares.

10. Collados Aís, A., Fernández Sánchez, M.M. y Gile, D. (eds.) (2003). La evaluación de la calidad en interpretación: investigación. Granada: Comares.

11. Falbo, C. (1998). “Analyse des erreurs en interprétation simultanée”. The Interpreters’ Newsletter 8, 105-120.

12. Fernández Sánchez, M.M. et al. (2007). "La incidencia del parámetro transmisión correcta del discurso original”. En Collados Aís, Á. et al. (eds.), 89-104. Granada: Comares.

13. Garzone, G. (2003). "Reliability of quality criteria evaluation in survey research”. En Collados Aís, A., Fernández Sánchez, M.M. \& Gile, D., 23-30. 
14. Gerver, D. (1974). "The effects of noise on the performance of simultaneous interpreters: Accuracy of performance”. Acta psychologica 38, 159-167.

15. Gerver, D. y Sinaiko, W. (1978). Language, interpretation and communication. Nueva Yor: Plenum.

16. Gile, D. (1983). “Aspects méthodologiques de l'évaluation de la qualité du travail en interprétation simultanée” Meta, 28: 3, 236-243.

17. Gile, D. (1990). “L’evaluation de la qualité de l'interprétation par les délégués : Une étude de cas ». The interpreters’ Newsletter 3, 66-71.

18. Gile, D. (1995). "Fidelity assessment in consecutive interpretation: An experiment”. Target, 7: 1, 151-164.

19. Gracia García, R. (2003). "Evaluadores potencias de la calidad en la interpretación simultánea de conferencias y la evaluación desde el punto de vista del oyente”. En Collados Aís, Á. et al. (eds.), 297-306. Granada: Comares.

20. Haensch, G. (1965). Técnica y picardía del intérprete diplomático. Múnich: Max Hueber Verlag.

21. Iglesias Fernández, E. (2007). La didáctica de la interpretación de conferencias. Granada: Comares.

22. Kopczynski, A. (1994). "Quality in conference interpreting: some pragmatic problems”. En Lambert, S. y Moser Mercer, B. (eds.), 8999.

23. Kurz, I. (1989). “Conference interpreting user expectations”. En Hammond, D (ed.). Coming of Age. Medford, N.J: Learned information, 143-148.

24. Kurz, I. (1993). “Conference interpreting: Expectations of different user groups”. The Interpreters' Newsletter 5, 13-21.

25. Kurz, I. (2003). "Conference interpreting: Quality in the ears of the users”. Meta 45:2, 394-409.

26. Lambert, S. y Moser Mercer, B. (eds.) (1994). Bridging de gap. Empirical research in the teaching of simultaneous interpreting. Ámsterdam/Filadelfia: John Benjamins.

27. Mahyub Rayaa, B. y M. Zarrouk, (2013). Interpretación Simultánea (Árabe-Español). método para la enseñanza-aprendizaje, Toledo: Escuela de Traductores de Toledo.

28. Mahyub Rayaa, B. y M. Zarrouk, (2017). A Handbook for Simultaneous Interpreting Training from English, French and Spanish to Arabic. Toledo: Escuela de Traductores de Toledo.

29. Mahyub Rayaa, B. (2013). “La interpretación simultánea árabeespañol y sus peculiaridades: docencia y profesión. Estudio piloto”. En O. García Becerra, E.M. Pradas Macías y R. Barranco-Droege 
(eds.). Quality in interpreting: widening the scope, 337-358. Granada: Comares.

30. Mahyub Rayaa, B., (2015). La interpretación simultánea árabeespañol y sus peculiaridades. Docencia y profesión, Granada: Universidad de Granada. Tesis doctoral.

31. Martin, A. (1999). "La enseñanza de la interpretación de conferencia”. En A. Gil de Carrasco y Leo Hickey (coords.). Aproximación a la Traducción. Madrid: Instituto Cervantes.

32. Pradas Macías, E.M. (2003). Repercusión del intraparametro pausas silenciosas en la fluidez: Influencia en las expectativas y en la evaluación de la calidad en interpretación simultánea. Tesis Doctoral. Universidad de Granada.

33. Pradas Macías, E.M. (2004). La fluidez y sus pausas: Enfoque desde la interpretación de conferencias. Granada: Comares.

34. Pradas Macías, E.M. et al. (2007). "La incidencia del parámetros transmisión completa del discurso original”. En Collados Aís, Á. et al. (eds.), 297-306. Granada: Comares.

\section{Anexos}

\section{Cuestionario de evaluación}

Para la aplicación de este cuestionario y la recogida de los datos, hemos contado con la inestimable colaboración de Abderrahman Haddad, en el marco de su trabajo de fin de grado (Traducción e Interpretación, especialidad Árabe).

Sexo: $\square$ Hombre $\square$ Mujer

Edad:

Cuál es su/s lengua/s A:

B:

C:

En caso de que sea un dialecto del árabe, indique cuál:

¿Cursa o ha cursado estudios de Traducción e Interpretación? $\square$ Sí $\square$ No

En caso afirmativo, indique en qué curso se encuentra: 


\section{DATOS PERSONALES}

\section{PREGUNTAS ACERCA DE LA INTERPRETACIÓN}

1. ¿Cómo calificaría la interpretación que ha oído?

\begin{tabular}{ll}
$\square$ & Muy bien \\
$\square$ & Bien \\
$\square$ & Regular \\
$\square$ & Mal \\
\hline$\square$ & Muy mal
\end{tabular}

\section{Justifique por qué:}

2. ¿Cómo calificaría la transmisión del mensaje por parte del intérprete?

$\square$ Muy correcta
$\square$ Correcta
$\square$ Poco correcta
$\square$ Muy incorrecta

\section{Justifique por qué:}

3. ¿Cree que el intérprete ha sabido hacer llegar todo el sentido del mensaje original?

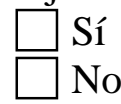

\section{Justifique su respuesta:}

4. ¿Cómo calificaría el español empleado por el intérprete en este caso?

\begin{tabular}{ll}
$\square$ & Muy bueno \\
$\square$ & Bueno \\
\hline$\square$ & Regular \\
\hline$\square$ & Malo \\
\hline$\square$ & Muy malo
\end{tabular}

\section{Justifique por qué:}

5. ¿Cree que el intérprete ha usado el registro apropiado para este tipo de discursos?

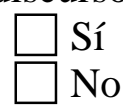

\section{Justifique su respuesta:}

6. ¿Cómo calificaría el vocabulario empleado por el intérprete?
$\square$ Muy correcto
$\square$ Correcto
$\square$ Poco correcto
$\square$ Muy incorrecto 


\section{$\square$ Otro. Especifique:}

7. ¿ ¿Le ha dificultado la comprensión del mensaje alguno de los aspectos de uso del español arriba mencionados (registro, vocabulario, estructura, etc.)?

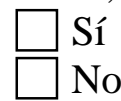

\section{Especifique cuál y justifique su respuesta:}

8. ¿Cree que los aspectos de uso del español arriba mencionados (registro, vocabulario, estructura, gramaticalidad, etc.) afectan la calidad de la interpretación?

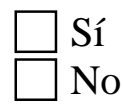

\section{Especifique cuál y justifique su respuesta:}

9. Califique la interpretación de 1 a 10 (donde 1 es "muy mal” y 10 "muy bien").
$1 \square 2$
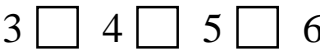
$6 \square 7$

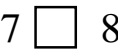
$8 \square 9$
$9 \square 10$

¡MUCHÍsImAs gRACIAS POR SU COLABORACIÓN! 